\title{
Silver and Copper nano-colloid generation via Pulsed Laser Ablation in Liquid: Recirculation nanoparticle production mode
}

Sithara Sreenilayam Pavithran, Ronan McCann, Éanna McCarthy, Brian Freeland, Karsten Fleischer, Stephen Goodnick, Stuart Bowden, Christiana Honsberg and Dermot Brabazon

Sithara Sreenilayam Pavithran. I-Form, Advanced Manufacturing Research Centre, \& Advanced Processing Technology

Research Centre, School of Mechanical and Manufacturing Engineering, Dublin City University, Glasnevin, Dublin-9, Ireland.

Corresponding author: Sithara.sreenilayam@dcu.ie

Ronan McCann. I-Form, Advanced Manufacturing Research Centre, \& Advanced Processing Technology Research Centre, School of Mechanical and Manufacturing Engineering, Dublin City University, Glasnevin, Dublin-9, Ireland.

Éanna McCarthy. I-Form, Advanced Manufacturing Research Centre, \& Advanced Processing Technology Research Centre, School of Mechanical and Manufacturing Engineering, Dublin City University, Glasnevin, Dublin-9, Ireland.

Brian Freeland. I-Form, Advanced Manufacturing Research Centre, \& Advanced Processing Technology Research Centre, School of Mechanical and Manufacturing Engineering, Dublin City University, Glasnevin, Dublin-9, Ireland.

Karsten Fleischer. I-Form, Advanced Manufacturing Research Centre, \& Advanced Processing Technology Research Centre, School of Mechanical and Manufacturing Engineering, Dublin City University, Glasnevin, Dublin-9, Ireland.

Stephen Goodnick. Quantum Energy for Sustainable Solar Technology (QESST) Engineering Research Center, Arizona State University, Tempe AZ 85281, United States.

Stuart Bowden. Quantum Energy for Sustainable Solar Technology (QESST) Engineering Research Center, Arizona State University, Tempe AZ 85281, United States.

Christiana Honsberg. Quantum Energy for Sustainable Solar Technology (QESST) Engineering Research Center, Arizona State University, Tempe AZ 85281, United States.

Dermot Brabazon. I-Form, Advanced Manufacturing Research Centre, \& Advanced Processing Technology Research Centre,

School of Mechanical and Manufacturing Engineering, Dublin City University, Glasnevin, Dublin-9, Ireland.

Abstract. Metal nanoparticles have unique chemical, physical, electrical, and optical properties that make them attractive for a wide range of applications in sensing, anti-fouling surfaces, medicine, and conductive inks. Pulsed Laser Ablation in Liquid (PLAL) is a green method of nanoparticle colloid production, capable of producing ligand-free nanoparticles in solution without the need for hazardous, environmentally unfriendly chemicals. Control of the process parameters can give control over the resulting colloid properties such as particle size distribution. In this work, silver (Ag) nanoparticles (NPs) with average particle size from 2.04 to $19.3 \mathrm{~nm}$ and copper $(\mathrm{Cu})$ NPs with average particle size from 40 to $85.9 \mathrm{~nm}$ were produced by PLAL) technique.

Keywords. Nanoparticle, Laser Ablation, Particle Size Distribution, Ablation Efficiency

\section{Introduction}

In recent years, conductive inks have received significant interest due to their application and popularity in printed electronics (PE) devices [1]. Printed electronics have a number of attractive attributes, such as lower costs, light weights, optical transparency, compatibility with flexible substrates and large scale production of devices on variety of different flexible substrates [2,3]. PE technology plays a key role in enabling extensive flexible and stretchable electronic devices. Nanomaterials including metal nanowires (NWs), [4,5] metal nanoparticles (NPs) [6,7], 2D nano materials (e.g., graphene [8,9], MXene [10,11]) and carbon (e.g., carbon nanotubes (CNTs) [12,13] are promising suitable candidates for conductive PEs. In recent years, much advancements on PE technologies based on nanomaterials, conductive ink generation, post printing processes such as sintering of printed tracks, and functional device integration have been reported [2]. Conductive patterns have been successfully applied to flexible substrates, paper and polymer, 
Silver and Copper nano-colloid generation via Pulsed Laser Ablation in Liquid: Recircul...

using conductive inks $[2,14,15]$. Nanomaterial-based conductive inks, made up of nanoscale pieces of conductive material in a liquid medium, are one option for PE devices. These nanomaterial inks are liquid at room temperature allowing them to be easily handled, processed, and deposited. Nanomaterial inks are commonly used in PE technology, using conductive nanomaterials such as silver (Ag) NPs [16], Ag NWs [17], copper (Cu) NPs [18], and CNTs [19, 20]. In recent years, the global conductive inks market has grown significantly due to their application in flexible PEs. Most commonly used functional conductive ink in the flexible PEs is Ag. Even in the oxide state, the Ag material conducts well and therefore the technological risk is low for this materials usage. If the conductivity of the ink is high, only less amount of ink is required for PE device production.

Nanoparticle production techniques are categorized as physical and chemical methods. Chemical reduction by inorganic and organic reducing agents or additives is the commonly used chemical method for NP production. Chemical technique use reducing agents to generate ions and atoms from the chosen precursor whereas, physical method normally uses laser impulse energy to reduce chosen bulk material into ions and atoms. The most important physical techniques are laser ablation and evaporation condensation. Pulsed Laser Ablation in Liquid (PLAL), sometimes given the name Laser Ablation Synthesis in Solution (LASIS), is a physical method of producing nanomaterials in solution. In this method, a pulsed laser is focused on the surface of a solid target in a liquid medium. Since the use of pulsed laser for generating metal nano colloidal solutions in different solvents by ablating metal target surfaces that immerse in the chosen solvent in 1993 by Henglein [21] and Cotton [22], the PLAL method has been extensively used to produce polymer [23], carbon [24,25], semiconductor (e.g., ZnO [26], Si [27], and SiC [28]) and metal (e.g., Au [29], Cu [30], and Ag [31]) NPs in liquid. In this technique laser energy absorbed by the target generates a plasma, which expands and condenses in the liquid environment, forming nanoscale pieces of material as the cavitation bubble expands and collapses. This method can produce ligand-free NPs, and generally doesn't require chemical agents beyond the solvent medium, making the method ecofriendly. The morphology of the NPs produced can be controlled by altering the process parameters such as solvent type, laser power, wavelength, and repetition rate. Using this PLAL technique, nanomaterials can be produced with high purity under normal pressure and temperature conditions. The NP size, their stability in liquid, and NP productivity can easily be controlled in this simple technique by changing the experimental parameters such as laser energy, laser wavelength, repetition rate, solvent etc. [32]. The physicochemical characteristics of nano colloidal solutions strongly affects the rate of NP formation, their particle size and shape and their polydispersity [33]. In the past decades, PLAL method has been widely studied for generating nanomaterials in colloidal solutions as this leads to the production of cost effective PE conductive inks.

Most of the nano colloidal generation via PLAL is static, i.e., the laser ablation of the surface of the chosen target performs within a container of liquid $[24,25]$. Although this static technique is simple, main limitation of this method is its low productivity which inhibit its acceptance in manufacturing industry [34]. In PE application the NP productivity plays a key role as the quality and conductivity of the printed track depends on the concentration and sizes of NPs in the colloids. Therefore, the present paper focuses on the laser ablation of $\mathrm{Ag}$ and $\mathrm{Cu}$ in deionized (DI) water in a dynamic recirculation production mode aiming to produce nano colloids with high efficiency for application such as inkjet printing. Here in this technique, the laser ablation occurs on the surface of the target material that immersed in a dynamic liquid. The influence of the process duration on the particle size distribution and production rate was investigated for both $\mathrm{Ag}$ and Cu materials. The average particle size distribution for the produced $\mathrm{Ag}$ and Cu NP colloids are in the range suitable for inkjet printing applications.

\section{Experiment}

Nano-colloids were generated using a picosecond, Nd:YAG laser (WEDGE HF 1064, BrightSolutions, Italy) by irradiating laser energy on the $\mathrm{Ag}$ and $\mathrm{Cu}$ target surfaces. The laser beam power was $1.44 \mathrm{~W}$. The target materials used were $\mathrm{Ag}$ and $\mathrm{Cu}(99.99+\%$ purity, sourced from Goodfellow Cambridge Ltd). The laser beam was rastered using a 2D scanning 
galvanometer (Raylase SS-12) at variable linear speeds moving in an Archimedean spiral pattern across the surface of the chosen target. A recirculation production mode set up with $100 \mathrm{ml} / \mathrm{min}$ liquid flow rate was used for nano colloids production. A flow-cell which is made using a Stratasys Connex1 3D printer with dual material print with VeroWhitePlus (RGD835) photopolymer (65 shore D) was used for keeping substrates and to produce nano-colloids, in DI water, in the recirculation NP production mode (Fig. 1).

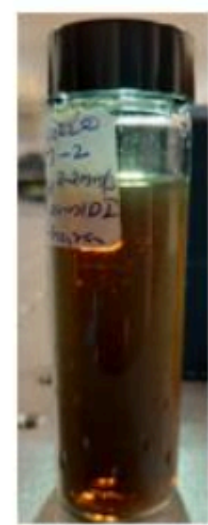

Silver

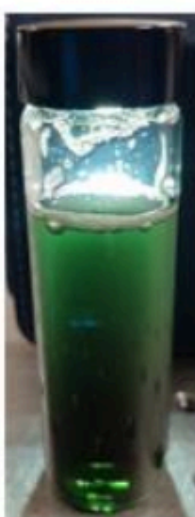

Copper

Nano-colloid Nano-colloid

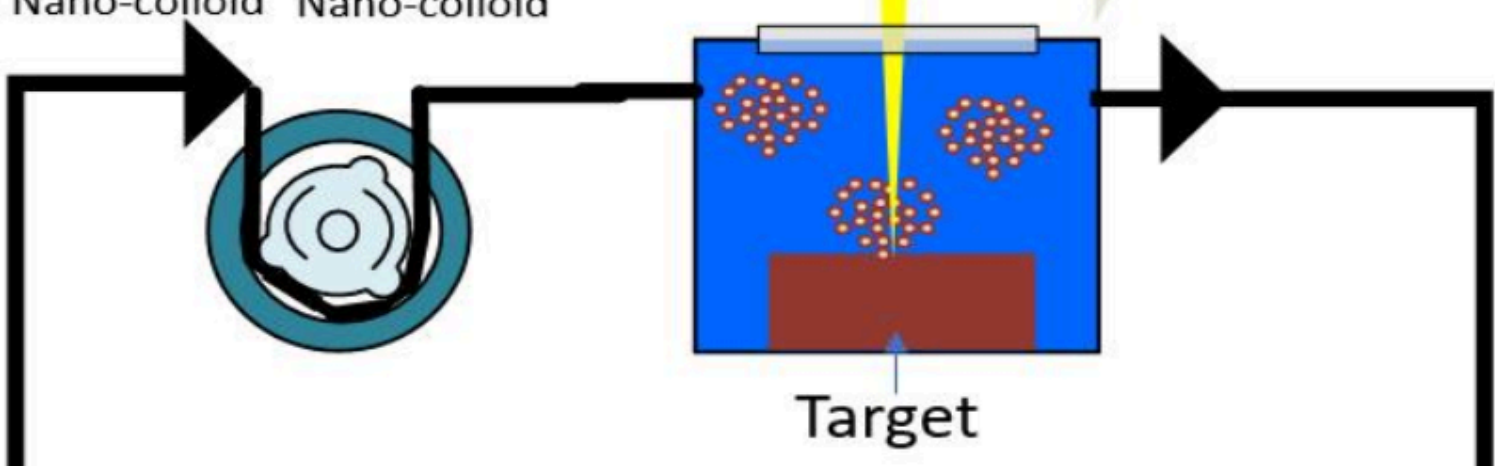

\section{Solvent Flow}

Fig. 1 Schematic of the recirculation production mode for the generation of NP colloids with the images of generated Ag and Cu NP colloids.

In this production method, $20 \mathrm{ml}$ of DI water recirculate through a closed-loop piping system and the target material that immersed in a flow cell, for a particular set time. The produced nano colloids were characterized using Dynamic Light Scattering, DLS (NANO-flex® $180^{\circ}$ DLS Size, Microtrac Ltd.), and UV-Vis spectroscopy (Libra S22 UV-Vis Spectrophotometer (Biochrom Inc., USA). The NPs productivity in DI water is estimated by evaluating the loss of Ag or $\mathrm{Cu}$ target mass before and after the laser ablation.

\section{Results and Discussions}

\subsection{Production of Silver (Ag) Nano colloids}

Ag NP solutions were produced by laser ablation of a solid target in DI water by varying target ablation time. Figure 
Silver and Copper nano-colloid generation via Pulsed Laser Ablation in Liquid: Recircul...

2 (a) shows the UV-Vis absorption spectra of the obtained colloidal solutions. All absorption spectra show the peaks in the $\sim 400 \mathrm{~nm}$ wavelength region. The location of this peak indicates metallic Ag NPs have been produced [35].The optical properties of Ag NPs are dependent highly on the NP diameter. Nano colloids consist of smaller particle size distribution absorb the incident light and exhibits absorption peaks around $400 \mathrm{~nm}$ wavelength region. However, the colloids consist of larger particle size distribution creates light scattering and form broad absorption peak that shift towards the longer wavelength region, is known as, red shifting. The optical properties of Ag NPs change when NPs aggregates. When this happens, the UV-Vis spectra shows shift of the surface plasmon resonance to lower energies leading the scattering and absorption peaks to red shift. The characterization method can be used as reliable and simple technique for recording the NP stability in solution. The intensity of the original peak will decrease with the depletion of the stable NPs and as a result either broad or secondary peaks formation occurs at longer wavelength region. UV/Visible spectroscopy can be used to characterize NP stability in a colloid over time.

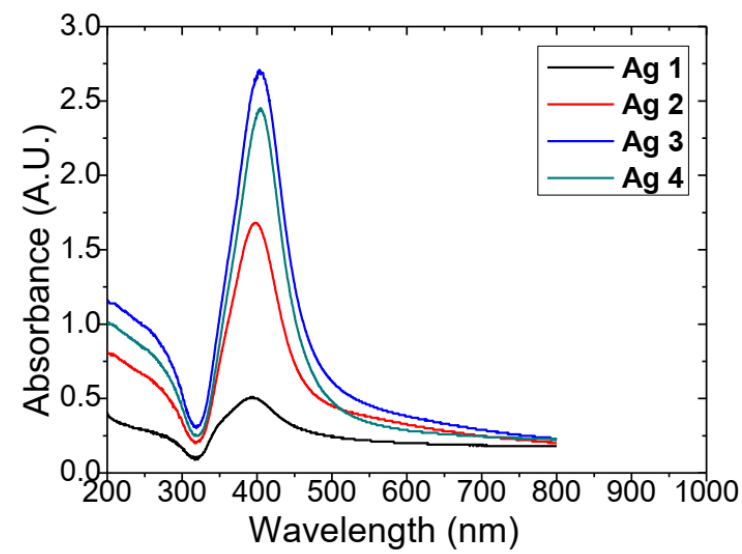

(a)
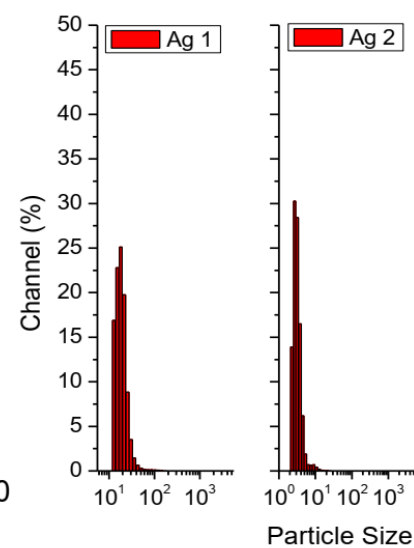

Particle Size Distribution $(\mathrm{nm})$

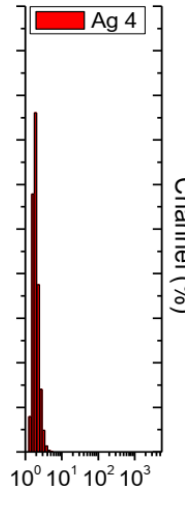

(b)

Fig. 2 (a) UV-Vis absorbance vs. wavelength (b) particle size distribution from DLS technique for the Ag NP colloids produced in the dynamic flow PLAL system with $100 \mathrm{ml} / \mathrm{min}$ liquid flowrate.

NP size distribution in colloidal solution can be determined by evaluating the random changes in the light intensity that scattered from the colloidal solution. For analyzing NPs after the production DLS technique is commonly used. In suspension, the smaller NPs undergo thermal random motion, which is called Brownian motion. This Brownian movement is modeled by the Stokes-Einstein Eq. As,

$$
D_{h}=\frac{k_{B} T}{3 \pi \eta D_{t}}
$$

where, $\mathrm{D}_{\mathrm{h}}=$ hydrodynamic diameter of NPs, $\eta=$ dynamic viscosity, $\mathrm{D}_{\mathrm{t}}=$ translational diffusion coefficient, $\mathrm{kB}=$ Boltzman's constant and $\mathrm{T}=$ thermodynamic temperature. Here, temperature is an important parameter consideration due to the presence of the term viscosity as the viscosity is a stiff function of temperature. Most importantly, it explains the particle size analyses from the DLS is the hydrodynamic size.

The DLS NP size distribution plots of the generated Ag NP colloids are shown in Fig. 2 (b). The colloidal solutions exhibit singular peak distribution with the particle size diameter between $2.04 \mathrm{~nm}$ to $19.3 \mathrm{~nm}$. 


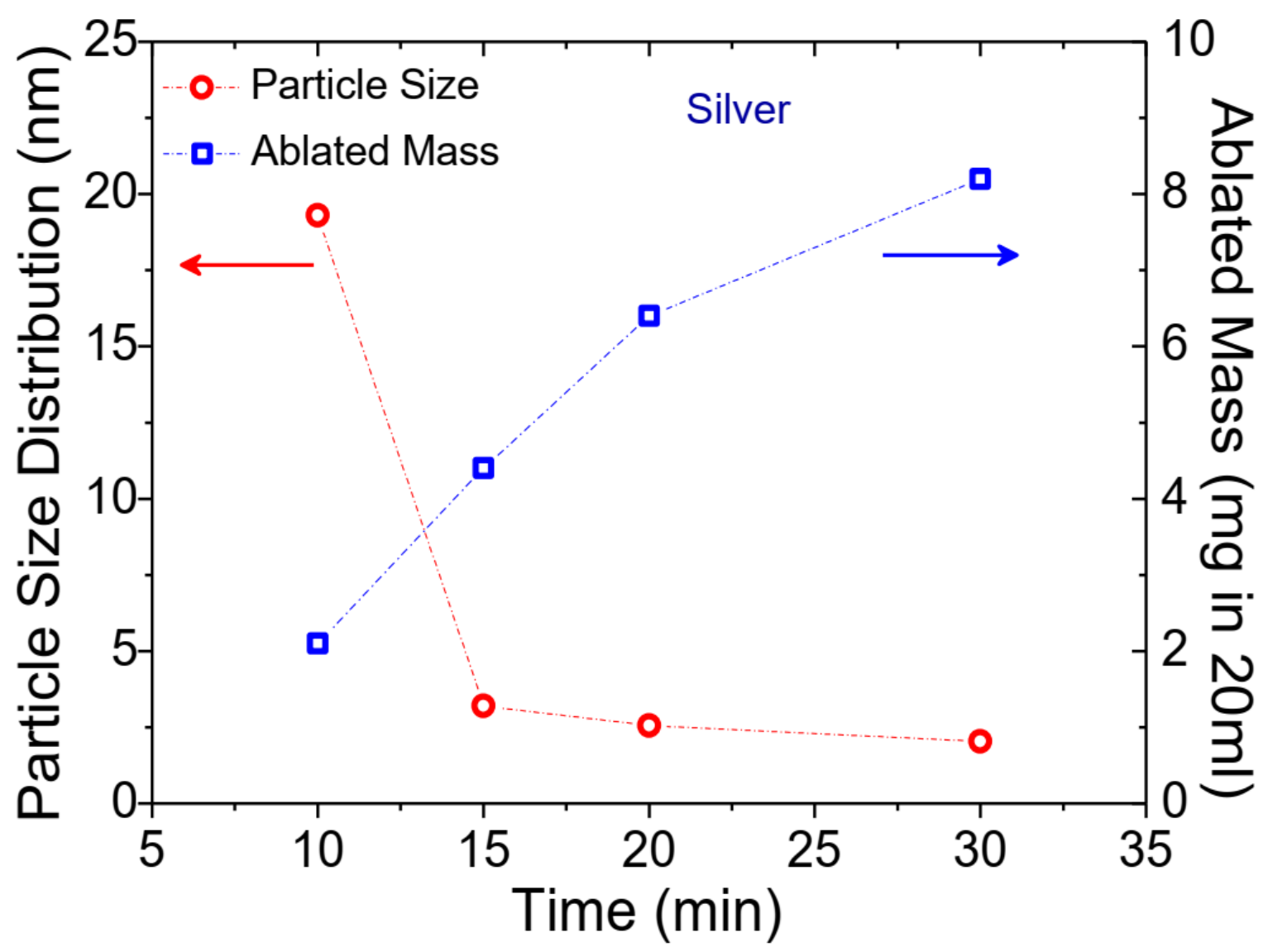

Fig. 3 Average particle size distribution and ablated mass for the Ag NP colloids produced in the dynamic flow PLAL system.

With an increase in the duration of laser ablation (Fig. 3), the yield of ablated Ag mass increases from $2.1 \mathrm{mg}$ (10 min) to $8.2 \mathrm{mg}$ (30 $\mathrm{min}$ ) in $20 \mathrm{ml}$ DI water. However, average particle size distribution shows a decreasing trend from 19. 3 $\mathrm{nm}(10 \mathrm{~min})$ to $2.04 \mathrm{~nm}$ (30 min). Table 1 list the details of the fixed and varied parameters used for Ag PLAL with the outcomes of ablated mass and the average particle size distribution.

Table 1. Details of PLAL parameters, NP average size distribution, and ablated mass of Cu NPs in $20 \mathrm{ml}$ DI water.

\begin{tabular}{|c|c|c|c|c|c|c|}
\hline Target & $\begin{array}{c}\text { Time } \\
{[\mathbf{m i n}]}\end{array}$ & $\begin{array}{c}\text { Repetition Rate } \\
{[\mathbf{k H z}]}\end{array}$ & $\begin{array}{c}\text { Laser } \\
\text { Power } \mathbf{( \% )}\end{array}$ & $\begin{array}{c}\text { Scan Speed } \\
(\mathbf{m m} / \mathbf{s})\end{array}$ & $\begin{array}{c}\text { Average NP Size } \\
(\mathbf{n m})\end{array}$ & $\begin{array}{c}\text { Ablated Ag mass in 20ml } \\
\text { DI water }(\mathbf{m g})\end{array}$ \\
\hline $\mathrm{Ag} 1$ & 10 & 10 & 100 & 2 & 19.3 & 2.1 \\
\hline $\mathrm{Ag} 2$ & 15 & 10 & 100 & 2 & 3.2 & 4.4 \\
\hline $\mathrm{Ag} 3$ & 20 & 10 & 100 & 2 & 2.56 & 6.4 \\
\hline $\mathrm{Ag} 4$ & 30 & 10 & 100 & 2 & 2.04 & 8.2 \\
\hline
\end{tabular}

\subsection{Production of Copper (Cu) Nano colloids}

The colloidal solutions of Cu NPs have been generated in DI water (see Fig. 1). The formation of Cu NPs in DI water directly detected due to the colour change of the solvent. The obtained colloidal solutions exhibit green color which indicate the formation oxidized $\mathrm{Cu}$. The UV-VIS absorbance spectra of the obtained $\mathrm{Cu}$ NP solutions are shown in Fig. 
Silver and Copper nano-colloid generation via Pulsed Laser Ablation in Liquid: Recircul...

4 (a). The absorption peaks are observed due to the surface plasmonic resonance of the Cu2O and CuO NPs. The absorption peaks observed between $200 \mathrm{~nm}-300 \mathrm{~nm}$ is due to the formation of CuO NPs and the peaks between 300 $\mathrm{nm}-400 \mathrm{~nm}$ corresponds to the $\mathrm{Cu}_{2} \mathrm{O}$ Nps.

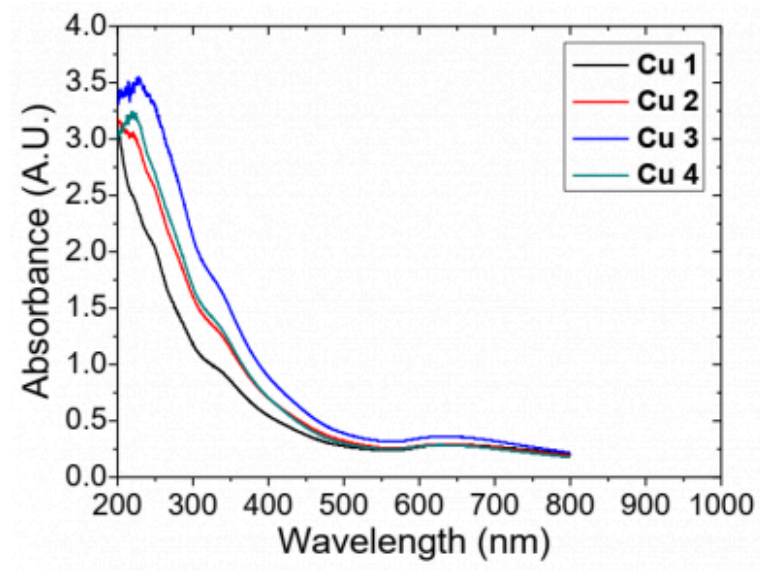

(a)

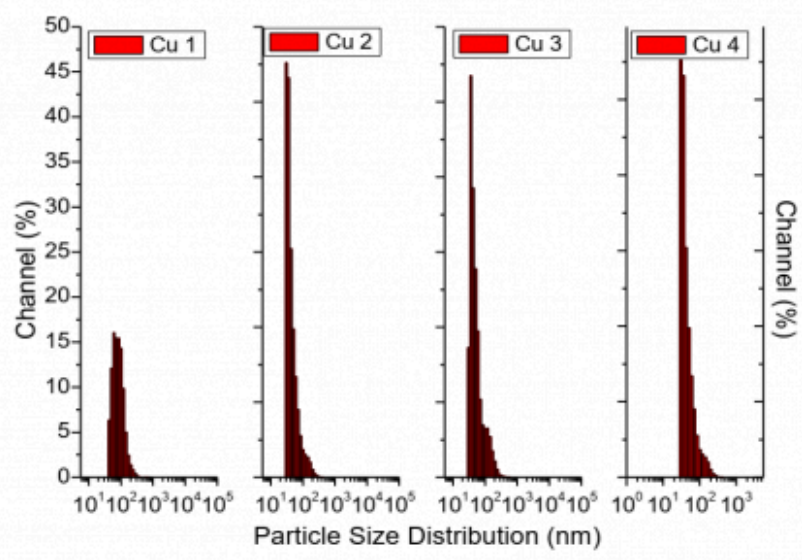

(b)

Fig. 4 (a) UV-Vis absorbance vs. wavelength (b) particle size distribution from DLS technique for the Cu NP colloids produced in the dynamic flow PLAL system with $100 \mathrm{ml} / \mathrm{min}$ liquid flowrate.

The DLS NP size distribution plots of the generated Cu NP colloids are shown in Fig. 4 (b). The colloidal solutions exhibit singular peak distribution with the particle size diameter between $40 \mathrm{~nm}$ to $90 \mathrm{~nm}$. With an increase in the duration of laser ablation (Fig. 5), the yield of ablated $\mathrm{Cu}$ mass increases from $2.4 \mathrm{mg}$ (15 min) to $4.8 \mathrm{mg}$ (45 min) in $20 \mathrm{ml}$ DI water, but on further increase in the duration of the laser ablation to 60 min shows a reduction in the ablated mass efficiency, i.e., $3.0 \mathrm{mg}$ in $20 \mathrm{ml}$ DI water. However, average $\mathrm{Cu}$ particle size distribution shows a decreasing trend from $85.9 \mathrm{~nm}$ (15 min) to $40 \mathrm{~nm}$ (60 min). 


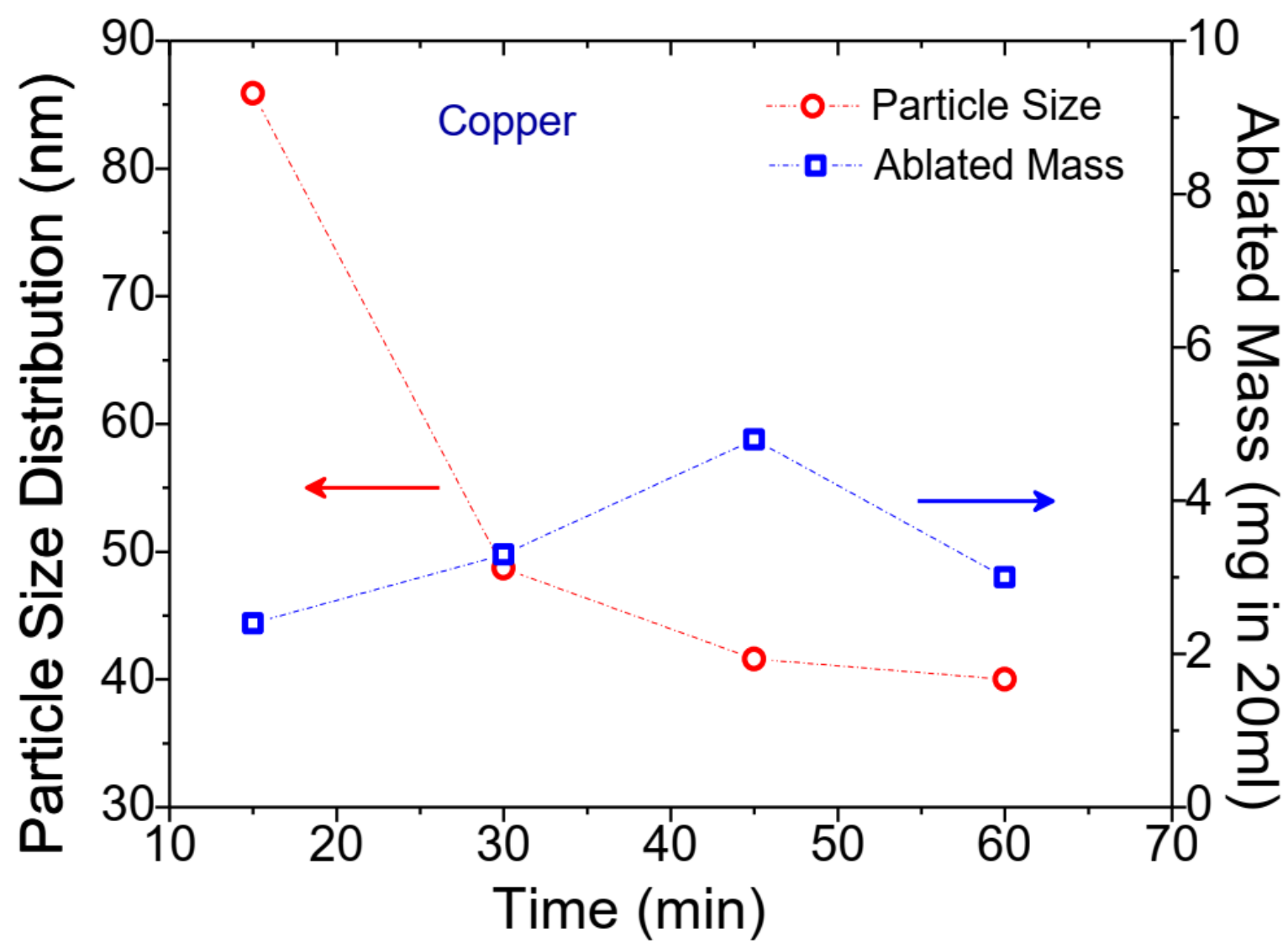

Fig. 5 Average particle size distribution and ablated mass for the Cu NP colloids produced in the dynamic flow PLAL system.

Table 2 list the details of the fixed and varied parameters used for Cu PLAL with the outcomes of ablated mass and the average particle size distribution.

Table 2. Details of PLAL parameters, NP average size distribution, and ablated mass of Cu NPs in 20 ml DI water.

\begin{tabular}{|c|c|c|c|c|c|c|}
\hline Target & $\begin{array}{c}\text { Time } \\
{[\mathbf{m i n}]}\end{array}$ & $\begin{array}{c}\text { Repetition } \\
\text { Rate }[\mathbf{H z}]\end{array}$ & $\begin{array}{c}\text { Laser } \\
\text { Power } \mathbf{( \% )}\end{array}$ & $\begin{array}{c}\text { Scan Speed } \\
(\mathbf{m m} / \mathbf{s})\end{array}$ & Average NP Size (nm) & $\begin{array}{c}\text { Ablated Cu mass in } \\
\text { 20ml DI water (mg) }\end{array}$ \\
\hline $\mathrm{Cu} 1$ & 15 & 10 & 100 & 2 & 85.9 & 2.4 \\
\hline $\mathrm{Cu} 2$ & 30 & 10 & 100 & 2 & 48.7 & 3.3 \\
\hline $\mathrm{Cu} 3$ & 45 & 10 & 100 & 2 & 41.6 & 4.8 \\
\hline $\mathrm{Cu} 4$ & 60 & 10 & 100 & 2 & 40 & 3.0 \\
\hline
\end{tabular}

The particle size and production rate of NPs produced in the DI water (20 ml) by laser ablation depends on the laser parameters, such as laser pulse energy and repetition rate, and the duration of the ablation. The highest concentration and NP production rate for $\mathrm{Cu}$ target was obtained for a 45-minute duration and for Ag target, it is 30-minute duration.

\section{Conclusions}

PLAL is an attractive method for producing nanomaterials in solution. Metal NPs in solution are suitable for use as conductive inks which can be used for PEs. The morphology, chemistry, and concentration of the NP colloids produced 
Silver and Copper nano-colloid generation via Pulsed Laser Ablation in Liquid: Recircul...

by PLAL are influenced by the process parameters. As such, the method can be optimized by varying these parameters to achieve the desired properties for a printable, conductive ink.

In this work, colloidal $\mathrm{Ag}$ and $\mathrm{Cu}$ NPs were successfully prepared by PLAL using a dynamic flow based system. The influence of the process duration on the particle size distribution and production rate was investigated for both materials. It was found that the average particle size decreased with process duration for both materials. This may be due to the re-circulation of the colloid throughout the process, with particles flowing back through the ablation site and being broken up by the laser. The ablated mass produced increased with duration as expected for the Ag NPs, with the rate of increase lowering at higher durations, possibly due to saturation of the colloid. For the copper target experiments, the ablation rate initially increased with duration, however the 60 min duration batch had a lower total mass produced than the 45-minute batch. Nanoparticles may sometimes deposit themselves onto the target after being produced, which could lead to a reduction in total mass [9]. However further reproducibility testing is needed in future work, to determine if this reduction in ablated mass happens consistently.

\section{Acknowledgements}

This work is supported in part by a research grant from Science Foundation Ireland (SFI) under Grant Numbers 16/ RC/3872, 19/US-C2C/3579, and is co-funded under the European Regional Development Fund.

\section{Bibliography}

[1] S. M. F. Cruz, L. A. Rocha, and J. C. Viana, "Printing Technologies on Flexible Substrates for Printed Electronics," in Flexible Electronics, IntechOpen, 2018.

[2] S.P. Sreenilayam, I. Ul Ahad, V. Nicolosi, V. Acinas, and D. Brabazon, "Advanced Materials of Printed Wearables for Physiological Parameter Monitoring," Materials Today, vol. 32, pp. 147-177, 2020.

[3] L. Nayak, S. Mohanty, S. K. Nayak, and A. Ramadoss, "A review on inkjet printing of nanoparticle inks for flexible electronics," J. Mater. Chem. C, vol. 7, no. 29, pp. 8771-8795, 2019.

[4] S. Yao, Y. Zhu, "Wearable multifunctional sensors using printed stretchable conductors made of silver nanowires", Nanoscale vol. 6, no. 4, pp. 2345-2352, 2014.

[5] M. Amjadi, A. Pichitpajongkit, S. Lee, S. Ryu, and I. Park, "Highly Stretchable and Sensitive Strain Sensor Based on Silver Nanowire-Elastomer Nanocomposite", ACS Nano, vol. 8, no. 5, pp. 5154-5163, 2014.

[6] Y. Hu, T. Zhao, P. Zhu, Y. Zhu, X. Shuai, X. Liang, R. Sun, D. D. Lu and C.-P. Wong, Low cost and highly conductive elastic composites for flexible and printable electronics", J. Mater. Chem. C, vol. 4, pp. 5839-5848, 2016.

[7] E.Skotadis, D.Mousadakos, K.Katsabrokou, S.Stathopoulos and D.Tsoukalas, "Flexible polyimide chemical sensors using platinum nanoparticles", Sensors and Actuators B: Chemical, vol. 189, pp. 106-112, 2013.

[8] D. J. Finn, M. Lotya, G. Cunningham, R. J. Smith, D. McCloskey, John F. Donegan and J. N. Coleman, "Inkjet deposition of liquid-exfoliated graphene and MoS2 nanosheets for printed device applications", J. Mater. Chem. C, vol., 2, pp. 925-932, 2014.

[9] K.-Y. Shin, J.-Y. Hong, and J. Jang, "Micro patterning of Graphene Sheets by Inkjet Printing and Its Wideband Dipole-Antenna Application", vol. 23, no. 18, pp. 2113, 2011.

[10] C. Zhang, L. McKeon, M. P. Kremer, S.-H. Park, O. Ronan, A. S.-Ascaso, S. Barwich, C. Ó Coileáin, N. McEvoy, H.C. 
Nerl, B. Anasori, J. N. Coleman, Y. Gogotsi and V. Nicolosi, "Additive-free MXene inks and direct printing of microsupercapacitors", Nat. Commun. vol. 10, no. 1795, pp. 1-9, 2019.

[11] S. P. Sreenilayam, I. Ul-Ahad, V. Nicolosi, and D. Brabazon, "MXene materials based Printed Flexible Devices for Healthcare, Bio-Medical and Energy Storage Applications", Materials Today, (in press), 2021.

[12] D. Son, J. H. Koo, J-K Song, J. Kim, M. Lee, H. J. Shim, M. Park, M. Lee, J. H. Kim, and D.-H. Kim, “Stretchable Carbon Nanotube Charge-Trap Floating-Gate Memory and Logic Devices for Wearable Electronics”, ACS Nano, vol. 9, no. 5, pp. 5585- 5593, 2015.

[13] L.-F. Ma, R.-Y. Bao, R. Dou, S.-D. Zheng, Z.-Y. Liu, R.-Y. Zhang, M.-B. Yang, W. Yang, “Conductive thermoplastic vulcanizates (TPVs) based on polypropylene (PP)/ethylene-propylene-diene rubber (EPDM) blend: From strain sensor to highly stretchable conductor", Composites Science and Technology, vol. 128, pp.176-184, 2016.

[14] Y. Gao, R. Liu, X. Wang, J. Liu, and Q. Fang, "Flexible RFID Tag Inductor Printed by Liquid Metal Ink Printer and Its Characterization,” J. Electron. Packag., vol. 138, no. 3, p. 031007, 2016.

[15] J. Matyas, L. Munster, R. Olejnik, K. Vlcek, and P. Slobodian, "Antenna of silver nanoparticles mounted on a flexible polymer substrate constructed using inkjet print technology Antenna of silver nanoparticles mounted on a fl exible polymer substrate constructed using inkjet print technology," vol. 13

[16] H. He, L. Sydänheimo, J. Virkki, and L. Ukkonen, “Experimental Study on Inkjet-Printed Passive UHF RFID Tags on Versatile Paper-Based Substrates," vol. 2016, pp. 1-9, 2016.

[17] N. Komoda, M. Nogi, K. Suganuma, K. Kohno, Y. Akiyama, and K. Otsuka, "Printed silver nanowire antennas with low signal loss at high-frequency radio," Nanoscale, vol. 4, no. 10, p. 3148, 2012.

[18] L. Sydanheimo, J. Virkki, L. Ukkonen, and Y. Ren, “Optimisation of manufacturing parameters for inkjet-printed and photonically sintered metallic nanoparticle UHF RFID tags," Electron. Lett., vol. 50, no. 21, pp. 1504-1505, 2014.

[19] B. Chen, Y. Jiang, X. Tang, Y. Pan, and S. Hu, "Fully Packaged Carbon Nanotube Supercapacitors by Direct Ink Writing on Flexible Substrates," ACS Appl. Mater. Interfaces, vol. 9, no. 34, pp. 28433-28440, 2017.

[20] A. De Giacomo et al., "Cavitation dynamics of laser ablation of bulk and wire-shaped metals in water during nanoparticles production," Phys. Chem. Chem. Phys., vol. 15, no. 9, pp. 3083-3092, 2013.

[21] A. Fojtik, A. Henglein, and B. Bunsen-Ges, "Laser vaporization of films and suspended particles in a solvent: formation of cluster and colloid solutions", Phys. Chem., vol. 97, pp. 252-254, 1993.

[22] J. Neddersen, G. Chumanov, T. M. Cotton, "Laser Ablation of Metals: A New Method for Preparing SERS Active Colloids", Appl. Spectrosc., vol. 47, pp. 1959-1964, 1993.

[23] D. E. Martínez-Tong, M. Sanz, T. A. Ezquerra, A. Nogales, J. F. Marco, M. Castillejo, E. Rebollar, “Formation of polymer nanoparticles by UV pulsed laser ablation of poly (bisphenol A carbonate) in liquid environment", Applied Surface Science, vol. 418, Part B, Pages 522-529, 2017.

[24] K Bagga, R McCann, M Wang, A Stalcup, M Vázquez and D Brabazon, "Laser assisted synthesis of carbon nanoparticles with controlled viscosities for printing applications", J Colloid Interface Sci., vol. 447, pp. 263-8, 2015.

[25] A. Al-Hamaoy, E. Chikarakara, H. Jawad, K. Gupta, D. Kumar, M.S. R. Rao, S. Krishnamurthy, M. Morshed, E. Fox, D. Brougham, X. He, M. Vázquez, D. Brabazon, "Liquid Phase - Pulsed Laser Ablation: A route to fabricate different carbon 
Silver and Copper nano-colloid generation via Pulsed Laser Ablation in Liquid: Recircul...

nanostructures", Applied Surface Science, vol. 302, pp. 141-144, 2014.

[26] H. B. Zeng, W. Cai, Y. Li, J. Hu, and P. Liu, “Composition/Structural Evolution and Optical Properties of ZnO/Zn Nanoparticles by Laser Ablation in Liquid Media”, J. Phys. Chem. B, vol. 109, no. 39, pp. 18260-18266 2005.

[27] M. M.-Carmona, and M. Vallet-Regí, "Advances in Laser Ablation Synthesized Silicon-Based Nanomaterials for the Prevention of Bacterial Infection", Nanomaterials, vol. 10, pp. 1443 (1-18), 2020.

[28] K. S. Khashan, R. A. Ismail, R. O. Mahdi, "Synthesis of SiC nanoparticles by SHG 532 nm Nd:YAG laser ablation of silicon in ethanol”, Applied Physics A, vol. 124, pp. 443 (1-11), 2018.

[29] F. Correard, K. Maximova, M.-A. Estève, C. Villard, M. Roy, A. Al-Kattan, M. Sentis, M. Gingras, A. V Kabashin, and D. Braguer, " Gold nanoparticles prepared by laser ablation in aqueous biocompatible solutions: assessment of safety and biological identity for nanomedicine applications" Int J Nanomedicine., vol. 9, pp. 5415-5430, 2014.

[30] M. T. Mohammed, A. A. Diwan, S. M. Saleh, and B. A. Salih, "Fabrication of copper nanoparticles by pulse laser ablation", Kufa Journal of Engineering, vol. 10, no. 1, pp. 1-11, 2019.

[31] M. Z. Alhamid, B. S. Hadi, and A. Khumaeni, "Synthesis of silver nanoparticles using laser ablation method utilizing Nd:YAG laser", AIP Conference Proceedings, vol. 2202, pp. 020013 (1-5), 2019.

[32] H. Zeng, X.-W. Du, S. C. Singh, S. A. Kulinich, S. Yang, J. He, and W. Cai, "Nanomaterials via Laser Ablation/ Irradiation in

Liquid: A Review” Advanced Functional Materials, vol. 22, no. 7, pp. 1333, 2012.

[33] M. Ocwieja, Z. Adamczyk, M. Morga, and K. Kubiak, "Silver particle monolayers — Formation, stability, applications" Adv

Colloid Interface Sci., vol. 222, pp. 530-563, 2015.

[34] R. Streubel, S. Barcikowski, and B. Gökce, "Continuous multigram nanoparticle synthesis by high-power, highrepetition-rate

ultrafast laser ablation in liquids," Opt. Lett., vol. 41, no. 7, p. 1486, 2016.

PDF automatically generated on 2021-05-25 10:20:24

Article url: https://popups.uliege.be/esaform21/index.php?id=2239

published by ULiège Library in Open Access under the terms and conditions of the CC-BY License (https://creativecommons.org/licenses/by/4.0) 